\title{
Unantimycin $A$, a new neoantimycin analog isolated from a microbial metabolite fraction library
}

\author{
Chung Liang Lim ${ }^{1,2,4}$, Toshihiko Nogawa ${ }^{1,4}$, Akiko Okano ${ }^{1}$, Yushi Futamura ${ }^{1}$, Makoto Kawatani ${ }^{1}$, \\ Shunji Takahashi ${ }^{3}$, Darah Ibrahim ${ }^{2}$ and Hiroyuki Osada ${ }^{1}$
}

The Journal of Antibiotics (2016) 69, 456-458; doi:10.1038/ja.2015.124; published online 9 December 2015

In the course of our screening for structurally unique secondary metabolites from a microbial metabolite fraction library by spectral database search, a new neoantimycin analog, unantimycin A (1) was discovered and isolated with a known neoantimycin analog, SW-163A (2). The structure of 1 was determined based on extensive spectroscopic methods, including NMR and MS. It had a 3-hydroxybenzoic acid moiety instead of a 2-hydroxy-3formylaminobenzoic acid moiety and showed moderate cytotoxicity against several cancer cell lines.

Microorganisms, such as actinomycetes and fungi, have a great capacity to produce a wide variety of structurally and biologically interesting secondary metabolites. ${ }^{1}$ They have been used for pharmaceutical drugs, agrochemicals and/or as leads. ${ }^{2,3}$ They are also important bioprobes, which are chemical tools to investigate biological functions in chemical biology studies. ${ }^{4,5}$ To discover and isolate such valuable metabolites, we have developed a methodology constructing a microbial metabolite fraction library combined with an original spectral database, named Natural Products Plot (NPPlot). ${ }^{6,7}$ The fraction library is composed of fractions, which are semi-purified extracts, prepared by basic chromatographic techniques, such as middle pressure liquid chromatography (MPLC) and HPLC, and some fractions contain an almost pure single metabolite. Each fraction is submitted to diode-array detector (DAD)-LC/MS analysis to collect physicochemical information including UV absorption and mass spectra of a metabolite within the fraction. This information is used to construct an NPPlot, which is a distribution map based on the physicochemical properties of each compound. These are plotted as dots on a 2D display with retention times and $m / z$ values on the $x$ and $y$ axes, respectively. On the basis of this methodology, we have discovered and isolated several new metabolites with unreported structures, such as verticilactam, ${ }^{8}$ spirotoamides ${ }^{9}$ and pyrrolizilactone. $^{10}$ New quinomycin derivatives, RK-1355A and B from Streptomyces sp. RK88-1355 were discovered using NPPlot screening, in which five NPPlots generated from different strains were compared and the distinctive metabolite group was identified as comprising isolated quinomycins. ${ }^{11}$ In our search for new metabolites by NPPlot screening of RK88-1355, another characteristic distribution was found in the region of retention time around $27 \mathrm{~min}$ with an $\mathrm{m} / \mathrm{z}$ value around 650 (Supplementary Figure S1). From this region, a new compound (1) was isolated together with a known neoantimycin analog, SW-163A $\mathrm{A}^{12}$ (2) from the related fractions by C18-HPLC. We report herein the isolation, structure and biological activities of $\mathbf{1}$ (Figure 1).

A 301 of culture broth of Streptomyces sp. RK88-1355 was used to construct the fraction library composed of $\sim 400$ fractions. The culture condition and construction of the library and NPPlot were described in the previous paper. ${ }^{11}$ The 34 th and 35th fraction of the second MPLC fraction was separated by C18-HPLC with acetonitrile/water (55:45) and (70:30) under isocratic elution to afford enriched fractions of compounds 2 and $\mathbf{1}$, respectively. The compound 1 rich fraction was further purified by C18-HPLC with isocratic elution of acetonitrile/water (58:42) to afford $\mathbf{1}(25.7 \mathrm{mg})$ as a colorless amorphous solid. The compound 2 rich fraction was purified by the same condition as that of $\mathbf{1}$ to afford $\mathbf{2}(3.3 \mathrm{mg})$ as a colorless amorphous solid. Compound 1: colorless amorphous solid; $[\alpha]_{589}{ }^{28}$ (c 0.1, $\left.\mathrm{CHCl}_{3}\right)-10.8^{\circ}$; UV (MeOH) $\lambda_{\max }(\log \varepsilon) 240$ sh (3.88), 290 (3.42) nm; IR (ATR) $\nu_{\max }\left(\mathrm{cm}^{-1}\right) 3370,2970,1750,1720,1650,1585$, $1520,1455,1190 ;$ HRESIMS $m / z 626.2589[\mathrm{M}+\mathrm{H}]^{+}$calcd for $\mathrm{C}_{33} \mathrm{H}_{40} \mathrm{NO}_{11}: 626.2601 ;{ }^{1} \mathrm{H}$ and ${ }^{13} \mathrm{C}$ NMR data were summarized in Table 1. Compound 2 was found to be identical with SW-163A ${ }^{12}$ by the comparison of their physicochemical properties including UV, IR, specific rotation, HRMS and NMR (described in Supplementary Information).

The molecular formula of $\mathbf{1}$ was determined to be $\mathrm{C}_{33} \mathrm{H}_{39} \mathrm{NO}_{11}$ by HRESIMS. The IR absorption spectrum implied the presence of ester (1750 and $1720 \mathrm{~cm}^{-1}$ ) and amide $\left(1650\right.$ and $1520 \mathrm{~cm}^{-1}$ ) groups. The ${ }^{1} \mathrm{H}$ NMR spectrum (Supplementary Figure S2) suggested that $\mathbf{1}$ was a compound related to 2 , containing six methyl groups and a benzene

${ }^{1}$ Chemical Biology Research Group, RIKEN Center for Sustainable Research Science, Wako, Saitama, Japan; ${ }^{2}$ Industrial Biotechnology Research Laboratory, School of Biological Sciences, Universiti Sains Malaysia, Minden, Penang, Malaysia and ${ }^{3}$ Natural Product Biosynthesis Research Unit, RIKEN Center for Sustainable Research Science, Wako, Saitama, Japan

${ }^{4}$ These authors contributed equally to this work.

Correspondence: Dr H Osada, Chemical Biology Research Group, RIKEN Center for Sustainable Research Science, 2-1 Hirosawa, Wako, Saitama 351-0198, Japan.

E-mail: hisyo@riken.jp

Received 10 August 2015; revised 4 November 2015; accepted 11 November 2015; published online 9 December 2015 


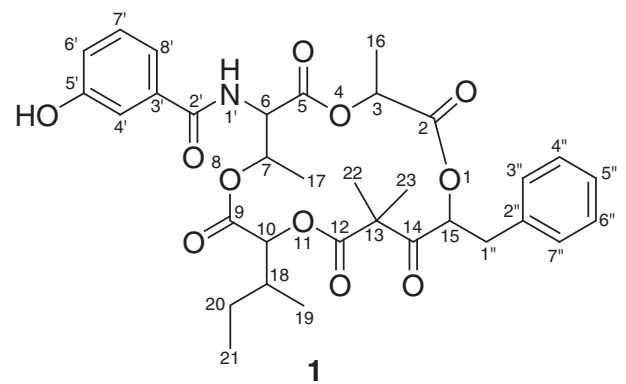<smiles>CCC(C)C(OC(=O)C(C)(C)C(O)C(Cc1ccccc1)OC(=O)C(C)OC(=O)C(NC(=O)c1cccc(NC=O)c1O)C(C)OC(=O)CO)C(C)CC</smiles>

Figure 1 Structures of compounds 1 and 2.

Table $1{ }^{1} \mathrm{H}$ and ${ }^{13} \mathrm{C}$ NMR chemical shifts of compound 1 in $\mathrm{CDCl}_{3}$

\begin{tabular}{|c|c|c|}
\hline Position & $\delta_{C}$ & $\delta_{H}($ multi, J in Hz) \\
\hline 2 & 169.1 & - \\
\hline 3 & 70.0 & $5.32(\mathrm{q}, 6.9)$ \\
\hline 5 & 168.5 & - \\
\hline 6 & 56.3 & $5.17(\mathrm{dd}, 9.2,2.9)$ \\
\hline 7 & 72.0 & $5.75(\mathrm{~m})^{\mathrm{a}}$ \\
\hline 9 & 168.5 & - \\
\hline 10 & 76.0 & $5.02(\mathrm{~d}, 9.2)$ \\
\hline 12 & 171.7 & - \\
\hline 13 & 54.0 & - \\
\hline 14 & 203.6 & - \\
\hline 15 & 79.2 & $5.76(\mathrm{~m})^{\mathrm{a}}$ \\
\hline 16 & 16.7 & $1.243(3 \mathrm{H}, \mathrm{d}, 6.9)$ \\
\hline 17 & 16.6 & $1.38(3 \mathrm{H}, \mathrm{d}, 6.3)$ \\
\hline 18 & 36.4 & $2.00(\mathrm{~m})$ \\
\hline 19 & 14.2 & $0.88(3 \mathrm{H}, \mathrm{d}, 6.9)$ \\
\hline \multirow[t]{2}{*}{20} & 24.6 & $1.14(\mathrm{~m})$ \\
\hline & & $1.49(\mathrm{~m})$ \\
\hline 21 & 10.5 & $0.87(3 \mathrm{H}, \mathrm{t}, 7.4)$ \\
\hline 22 & 20.7 & $1.236(3 \mathrm{H}, \mathrm{s})$ \\
\hline 23 & 22.7 & $1.40(3 \mathrm{H}, \mathrm{s})$ \\
\hline $11^{\prime}-\mathrm{NH}$ & - & $6.97(d, 9.2)$ \\
\hline $2^{\prime}$ & 168.2 & - \\
\hline $3^{\prime}$ & 135.0 & - \\
\hline $4^{\prime}$ & 115.0 & $7.49(\mathrm{dd}, 1.1,1.1)$ \\
\hline $5^{\prime}$ & 156.6 & - \\
\hline $6^{\prime}$ & 119.7 & $7.04(\mathrm{ddd}, 8.0,1.1,1.1)$ \\
\hline $7^{\prime}$ & 130.3 & 7.34 (dd, 8.0, 8.0) \\
\hline $8^{\prime}$ & 119.2 & $7.40(\mathrm{ddd}, 8.0,1.1,1.1)$ \\
\hline \multirow[t]{2}{*}{$1^{\prime \prime}$} & 37.7 & $3.13(\mathrm{dd}, 14.9,10.3)$ \\
\hline & & $3.38(\mathrm{dd}, 14.9,2.9)$ \\
\hline $2^{\prime \prime}$ & 136.6 & - \\
\hline 3", 7" & 129.6 & $7.29(\mathrm{~m})$ \\
\hline $4^{\prime \prime}, 6^{\prime \prime}$ & 128.7 & $7.30(\mathrm{~m})$ \\
\hline $5^{\prime \prime}$ & 127.2 & $7.23(\mathrm{~m})$ \\
\hline
\end{tabular}

$1 \mathrm{H}$ and $13 \mathrm{C} \mathrm{NMR}$ were recorded at 500 and $125 \mathrm{MHz}$, respectively. Exchangeable.

ring (7.23 p.p.m., $\mathrm{m}$ and $4 \mathrm{H}$ at $7.28-7.31$ p.p.m., m). However, it lacked the signals for 2-hydroxy-3-formylaminobenzoic acid moiety and showed the presence of a 1,3-disubstituted benzene moiety (7.49 p.p.m., dd, $J=1.1,1.1 \mathrm{~Hz}, 7.04$ p.p.m., ddd, $J=8.0,1.1,1.1 \mathrm{~Hz}$, 7.34 p.p.m., dd, $J=8.0,8.0 \mathrm{~Hz}$, and 7.40 p.p.m., ddd, $J=8.0,1.1$, $1.1 \mathrm{~Hz}$ ). The ${ }^{13} \mathrm{C}$ NMR spectrum (Supplementary Figure S3) was also similar to that of 2 and showed 31 signals including 10 aromatic signals, two of which at 128.7 and 129.6 p.p.m. had double the intensity of the others, supporting the presence of a mono- or

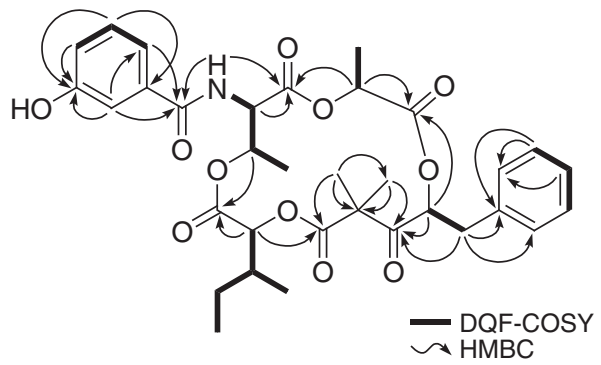

Figure 2 Key 2D NMR correlations of compound 1.

Table 2 Biological activities of compounds 1 and 2

\begin{tabular}{|c|c|c|c|}
\hline & 1 & 2 & Positive controp \\
\hline \multicolumn{4}{|l|}{ Cytotoxicity $\left(I_{50}: \mu M\right)$} \\
\hline HeLab & 12 & 14 & 0.0053 \\
\hline HL-60c & 6.7 & 16.0 & 0.00028 \\
\hline HT1080d & 11 & 5.7 & 0.0058 \\
\hline PANC- $1^{\mathrm{e}}$ & 22 & 24 & 0.020 \\
\hline$s r c^{\text {ts }}-N_{R K}^{f}$ & $>10$ & $>10$ & 2.6 \\
\hline VMRC-RCWg & 26 & 39 & 0.73 \\
\hline \multicolumn{4}{|l|}{ Antimicrobial activity $\left(I C_{50}: \mu M\right)$} \\
\hline Staphylococcus aureous 209 & $>50$ & $>50$ & 2.6 \\
\hline Escherichia coli $\mathrm{H} 0141$ & $>50$ & $>50$ & 0.87 \\
\hline Aspergillus fumigatus Af293 & $>50$ & $>50$ & 0.69 \\
\hline Pyricularia oryzae kita-1 & $>50$ & 0.034 & 0.10 \\
\hline Candida albicans JCM1542 & $>50$ & 0.12 & 0.034 \\
\hline Erythricium salmonicolor MAFF625123 & $>50$ & 1.5 & 0.74 \\
\hline \multicolumn{4}{|l|}{ Antimalarial activity $\left(I_{50}: \mu \mathrm{M}\right)$} \\
\hline Plasmodium falciparum 3D7 & 5.3 & 3.6 & 0.022 \\
\hline
\end{tabular}

aThe following compounds were used as positivie control for each assay: taxol for cytotoxicity test, chloramphenicol for anti-procaryote test, amphotericin B for anti-fungi test and chloroquine for anti-malarila test.

bHuman cervix epidermoid carcinoma.

cHuman promyelocytic leukemia.

dHuman sarcoma cell line with activated $\mathrm{N}$-ras oncogene.

eHuman pancreatic carcinoma.

Rat kidney cells infected with ts25, a T-class mutant of Rous sarcoma virus Prague strain.

gMalignant melanoma.

1,4-substituted benzene ring. The remaining six aromatic signals implied the sub-structure of an oxygen-substituted benzoic acid moiety in consideration of a low-field chemical shift value at 156.6 p.p.m. and the coupling pattern in ${ }^{1} \mathrm{H}$ NMR spectrum. This spectrum contained six methyl signals and four oxygenated signals. The latter were also evident in the ${ }^{13} \mathrm{C}$ NMR spectrum, which also contained six carbonyl signals including a ketone signal at 203.6 p.p.m. 
These assignments were confirmed by a DEPT experiment and an HSQC spectrum (Supplementary Figures S4 and S5). These observations suggested that $\mathbf{1}$ differed from $\mathbf{2}$ by having the macrocyclic ring alcohol oxidized to a ketone and attachment of 3-hydroxy-substituted benzoic acid instead of the 2-hydroxy-3-formylaminobenzoic acid. The overall structure was confirmed by the interpretation of DQF-COSY and HMBC spectra (Figure 2 and Supplementary Figures S6 and S7) and the structure of 1 was determined as shown in Figure 1, designated as unantimycin A.

Cytotoxic, antimicrobial and antimalarial activities of compounds $\mathbf{1}$ and 2 were evaluated in vitro. Compound 1 showed moderate cytotoxicity with $\mathrm{IC}_{50}$ values of $\sim 10 \mu \mathrm{M}$ against HeLa, HL-60 and HT1080 cell lines (Table 2). 1 also showed antimalarial activity with an IC $_{50}$ value of $5.3 \mu \mathrm{M}$, but did not show any effect on microbes up to $50 \mu \mathrm{M}$. Compound 2 on the other hand showed potent antifungal activities against Pyricularia oryzae and Candida albicans with $\mathrm{IC}_{50}$ values of 0.034 and $0.12 \mu \mathrm{M}$, which were similar to those of reported, ${ }^{12}$ and also against Erythricium salmonicolor with an $\mathrm{IC}_{50}$ value of $1.5 \mu \mathrm{M}$. The cytotoxicity and antimalarial activities were similar to those of 1, which suggested that the 2-hydroxy-3formylaminobenzoic acid moiety was essential for the antifungal activity.

Compound 1 has the 3-hydroxybenzoic acid instead of 2-hydroxy3 -formylaminobenzoic acid, which is the representative functional group for neoantimycin and antimycin classes of compounds. There have been reported some of neoantimycin analogs with different benzoic acid moiety, such as JBIR-04 containing a non-substituted benzene and JBIR-05, which lack the formyl group. ${ }^{13}$ However, antimycins or neoantimycins with a 3-hydroxybenzoate moiety has not been reported as a natural product to the best our knowledge. It is speculated that a 3-hydroxybenzoic acid instead of a 2-hydroxy-3formylaminobenzoic acid is mainly incorporated as the starter unit for the biosynthesis of 1 . The absolute configuration of neoantimycin analogs, SW-163A (2) and prunustatin A, which was oxidized derivative of 2 at $\mathrm{C}-14$ position as similar to the 15 -membered ring of 1, has been reported. ${ }^{14,15}$ Even though the NMR chemical shift values were almost identical with those of prunustatin A on the 15membered ring, except for the ${ }^{1} \mathrm{H}$ NMR chemical shift of $\mathrm{H}_{3}-16$ (prunustatin A: 1.43 p.p.m., 1: 1.243 p.p.m.), the specific rotation values were opposite (prunustatin $\mathrm{A}:+21.2^{\circ}\left(c 0.01, \mathrm{CHCl}_{3}\right), 1:-10.8^{\circ}$ $\left(c 0.1, \mathrm{CHCl}_{3}\right)$ ). Also, SW-163A (2) had the positive specific rotation value of $+63.7^{\circ}\left(c 0.1, \mathrm{CHCl}_{3}\right)$ (our experiment) $/+47.8^{\circ}$ (c 0.01 , $\mathrm{CHCl}_{3}$ ) (reported data ${ }^{12}$ ). The negative values were reported for JBIR-04 and $05\left(-28.6^{\circ}\right.$ (c 0.01, MeOH) and $-17.3^{\circ}$ (c 0.03, $\left.\mathrm{MeOH}\right)$, respectively). ${ }^{13}$ JBIR-04 had the same 15 -membered ring as that of $\mathbf{1}$ and the NMR chemical shift values were identical with those of $\mathbf{1}$ including ${ }^{1} \mathrm{H}$ NMR chemical shift value of $\mathrm{H}_{3}-16$. Therefore, the absolute configuration of $\mathbf{1}$ seemed to be different with those of prunustatin A and SW-163A (2) and related to those of JBIR-04 and 05. In the NPPlot, we have found some metabolites related to compound 1 , and will report these structures with consideration of stereochemistry in the near future. These findings suggest that our methodology of constructing a fraction library and using NPPlot to screen for novel structures is advantageous in efficiently discovering and isolating new metabolites.

\section{CONFLICT OF INTEREST}

The authors declare no conflict of interest.

\section{ACKNOWLEDGEMENTS}

We thank Dr Y Hongo in RIKEN for the HRESIMS measurements, Ms H Aono and Dr F Ito in RIKEN for activity tests, and Dr T Shimizu for valuable discussion about structures. This work was supported in part by JSPS KAKENHI Grant No. 24248022 and 26450148, and grant-in-aid from Research Program on Hepatitis from the Japan Agency for Medical Research and Development, AMED.

1 Osada, H. An overview on the diversity of actinomycete metabolites. Actinomycetol 15, 11-14 (2001).

2 Newman, D. J. \& Cragg, G. M. Natural products as sources of new drugs over the 30 years from 1981 to 2010. J. Nat. Prod. 75, 311-335 (2012).

3 Dobson, C. M. Chemical space and biology. Nature 432, 824-828 (2004).

4 Osada, H. in Bioprobes (ed. Osada, H.) 1-14 (Springer, Berlin, 2000).

5 Osada, H. Chemical biology based on small molecule-protein interaction. in Protein targeting with small molecules. Chemical biology techniques and applications (ed. Osada H.) 1-10 (Wiley, New Jersey, 2009).

6 Osada, H. \& Nogawa, T. Systematic isolation of microbial metabolites for natural products depository (NPDepo). Pure Appl. Chem. 81, 1407-1420 (2012).

7 Kato, N., Takahashi, S., Nogawa, T., Saito, T. \& Osada, H. Construction of a microbial natural product library for chemical biology studies. Curr. Opin. Chem. Biol. 16, 101-108 (2012).

8 Nogawa, T. et al. Verticilactam, a new macrolactam isolated from a microbial metabolite fraction library. Org. Lett. 12, 4564-4567 (2010).

9 Nogawa, T. et al. Spirotoamides A and B, novel 6,6-spitoaccetal polyketides isolated from a microbial metabolite fraction library. J. Antibiot. 65, 123-128 (2012).

10 Nogawa, T. et al. Pyrrolizilactone, a new pyrrolizidinone metabolite produced by a fungus. J. Antibiot. 66, 621-623 (2013).

$11 \mathrm{Lim}, \mathrm{C}$. L. et al. RK-1355A and B, novel quinomycin derivatives isolated from a microbial metabolites fraction library based on NPPlot screening. J. Antibiot. 67, 323-329 (2014).

12 Takahashi, K., Tsuda, E. \& Kurosawa, K. SW-163A and B, novel immunosuppressants produced by Streptomyces sp. J. Antibiot. 54, 867-873 (2001).

13 Izumikawa, M. et al. Novel GRP78 molecular chaperone expression down-regulators JBIR-04 and -05 isolated from Streptomyces violaceoniger. J. Antibiot. 60, 640-644 (2007).

14 Umeda, Y. et al. Absolute structure of prunustatin A, a novel GRP78 molecular chaperone down-regulator. Org. Lett. 9, 4239-4242 (2007).

15 Yamakoshi, S. \& Kawanishi, E. First total synthesis of prunustatin A. Tetrahedron Lett. 55, 1175-1177 (2014).

Supplementary Information accompanies the paper on The Journal of Antibiotics website (http://www.nature.com/ja) 